\title{
An exciting boost for solar cells
}

A process called singlet fission has the potential to enhance the efficiency of solar cells. The mechanism has been difficult to implement in such devices, but experiments demonstrate a way forward. SEE LETTER P.90

JOSEPH M. LUTHER

\& JUSTIN C. JOHNSON

$\mathrm{H}$ umans have been harnessing the enormous power of the Sun for millennia. The total amount of solar power received on Earth at any moment is about $10^{17}$ watts, whereas the global electricity demand is about $10^{12} \mathrm{~W}$ (see go.nature.com/2leb8sk). Although solar cells might seem ubiquitous, only $1.3 \%$ of electricity generated in the United States comes from these devices (see go.nature.com/2leb8sk). Therefore, the solar-cell industry still has immense opportunity for growth, to provide clean and renewable energy for the world's population. Economic factors drive decision-making in the energy sector, and thus, for greater adoption of solar cells, researchers are continually driven to make these devices more durable and more efficient at converting sunlight into electricity. On page 90, Einzinger et al. ${ }^{1}$ report a potential way to increase the efficiency of solar cells.

Improving the efficiency of highly optimized solar cells without introducing substantial complexity or cost is hard to achieve, but is a potentially transformative goal. Silicon solar cells currently dominate the market, but have welldocumented efficiency limitations. The most glaring deficiency, described in 1961, is that high-energy photons generate unwanted heat ${ }^{2}$. Conventional silicon solar cells are therefore restricted to converting only $30 \%$ of incident solar energy into electricity in an otherwise perfect device ${ }^{2}$. Strategies for overcoming this barrier include stacking different solar cells in series, or finding ways to split the energy of photons before collection.

Einzinger and colleagues demonstrate an approach by which a molecular layer splits a high-energy excitation that is generated by the absorption of a high-energy photon into two lower-energy excitations (Fig. 1). These excitations are subsequently transferred across a carefully designed interface into a silicon solar cell, where they are converted into an electric current. This strategy involves

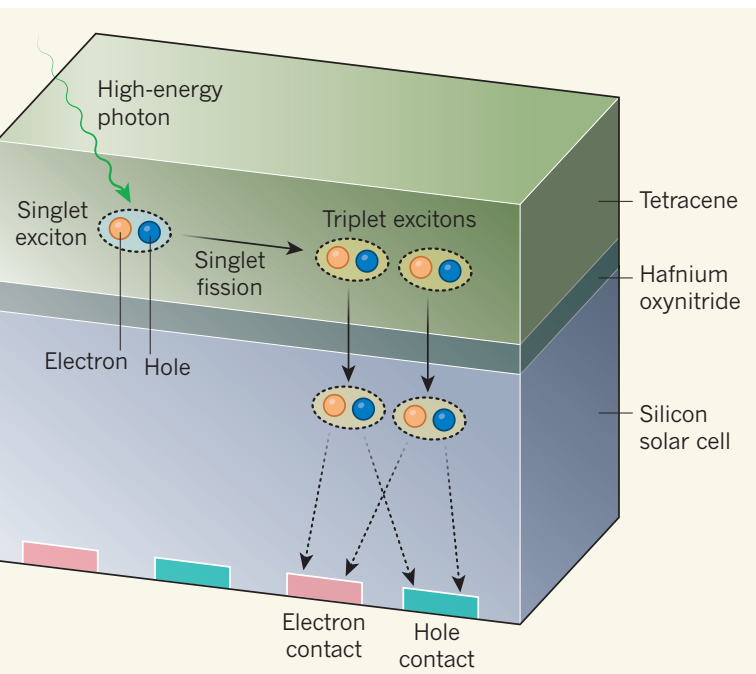

Figure 1 | A singlet-fission solar cell. Einzinger et al. ${ }^{1}$ report experiments on a silicon solar cell that has its top surface covered by n ultrathin coating of hafnium oxynitride. A layer of the materia igh-energy photon by the tetracene layer produces a singlet exciton, bound state of an electron and a hole (electron vacancy) that has zero pin (magnetic moment). This singlet exciton undergoes a process pairs that have a spin of 1 . These excitons are then transferred through 列 device, which gives rise to an electric current.

no extra electrical contacts or modification to the operation of the solar cell. It has long been considered a potentially simple yet effective way to avoid losing energy as heat and to design silicon solar cells that could have an efficiency of up to 1.4 times that of state-ofthe-art devices ${ }^{3,4}$

The process by which a pair of low-energy excitations is formed from one absorbed photon is called singlet fission. This mechanism has undergone a resurgence of interest largely because of its potential for enhancing solarenergy harvesting ${ }^{5-7}$. The archetypal material to enable singlet fission is called crystalline tetracene. This material splits (high-energy) blue or green light into two excitations that have energies similar to that of the lowestenergy excitation of silicon. Such energy matching makes tetracene and silicon ideal potential partners for the transfer of singletfission products. The combination of tetracene and a silicon solar cell was previously used to search for evidence of efficiency enhancement caused by singlet fission, but the crucial step of transferring the low-energy excitations into the solar cell could not be verified ${ }^{8,9}$.

In singlet fission, the high-energy excitation is a singlet exciton, a bound state of an electron and an electron vacancy (a hole) that has zero magnetic moment (that is, zero spin). The low-energy excitations are triplet excitons - electron-hole pairs that have a spin of 1 . Unlike their singlet counterparts, triplet excitons cannot return to the ground state by emitting radiation, because of quantum-mechanical spin-selection rules. This restriction elongates their lifetime but inhibits a common energy-transfer mechanism that would otherwise enable long-range transfer of the excitations through, for example, a thick passivating layer (an inert material that protects the surface of a solar cell from contamination).

In 1979, the physicist David Dexter recognized the potential use of a tetracene layer on top of a silicon solar cell ${ }^{3}$. He envisaged that two triplet excitons born from singlet fission could be transferred into the solar cell efficiently by a distinct mechanism that is common for these excitations, thereby doubling the electric current produced by the device for blue and green light absorbed from the Sun. However, this mechanism has a short range and can occur efficiently only through ultrathin barriers. Such barriers are often insufficient to passivate the surfaces of semiconductors such as silicon, resulting in power losses that could overwhelm the beneficial effects of singlet fission. Although the optical properties of tetracene are ideal for this energytransfer process, its poor electrical properties greatly complicate its direct integration into high-efficiency solar cells.

The remarkable demonstration presented by Einzinger et al. is based on the discovery that an exceptionally thin passivation layer can be made from hafnium oxynitride. This material can be deposited in such a way that its thickness is controlled with high precision and can 
protect the silicon surface at a thickness of only 0.8 nanometres. Conventional solar cells use opposing electrical contacts on the top and bottom of a light-absorbing semiconductor to extract electrons and holes, which gives rise to the electric current. By contrast, some high-efficiency solar cells are designed with both types of contact separated, but on the rear of the device. Such a rear-contact cell design, coupled with the passivation layer, is key to the authors' singlet-fission solar cell.

Einzinger and colleagues show that, when the tetracene layer on top of their solar cell is excited with blue or green light, triplet excitons are formed (Fig. 1). These excitons are transferred through the hafnium oxynitride into the silicon solar cell, without the need for extra electrical contacts. The exciton yield, which is defined as the average number of excitons transferred into the solar cell per photon, is about 1.3. In addition to the yield exceeding 1 , further evidence for triplet-exciton transfer lies in magnetic-field effects that have a distinctive signature for triplet excitons born from singlet fission.

After excitation of the tetracene, collisions between electrons and holes in the silicon layer lead to the emission of light. Modelling of this light shows that $76 \%$ of triplet excitons are transferred through the passivation layer, compared with $56 \%$ of singlet excitons. The singlet excitons are not beneficial because they represent only one electron-hole pair per photon. Therefore, reducing their influence on the solar cell by increasing the rate of singlet fission in the tetracene, and thus the proportion of triplets at the interface, is a major goal for the near future.

At this stage, Einzinger and colleagues' solar cell is relatively inefficient and the design is ripe for optimization. The hafnium oxynitride passivates the silicon surface, but the first few injected electrons and holes are found to initially fill imperfections at the silicon surface before moving into the solar cell. This finding indicates that the interface is still imperfect, but nevertheless shows the potential for this strategy in a working solar cell. Furthermore, the mechanism of triplet-exciton transfer, and how it can be expedited, is currently not described well by theory. Despite the need for considerable improvement, the field is now set on a route towards efficient triplet-exciton transfer into silicon that could some day make Dexter's dream a reality.

Joseph M. Luther and Justin C. Johnson are at the National Renewable Energy Laboratory, Golden, Colorado 80401, USA. e-mails: joey.luther@nrel.gov; justin.johnson@nrel.gov

1. Einzinger, M. et al. Nature 571, 90-94 (2019)

2. Shockley, W. \& Queisser H. J. J. Appl. Phys. 32, 510-519 (1961).

3. Dexter, D. L. J. Lumin. 18-19, 779-784 (1979)

4. Hanna, M. C. \& Nozik, A. J. J. Appl. Phys. 100, 074510 (2006).

5. Congreve, D. N. et al. Science 340, 334-337 (2013)
6. Paci, I. et al. J. Am. Chem. Soc. 128, 16546-16553 (2006)

7. Smith, M. B. \& Michl, J. Chem. Rev. 110, 6891-6936 (2010).

8. MacQueen, R. W. et al. Mater. Horiz. 5, 1065-1075 (2018)

9. Piland, G. B. et al. Chem. Phys. Lett. 601, 33-38 (2014).

\section{Metabolic signal curbs cancer-cell migration}

Metastasis, the migration of tumour cells from their primary site, is associated with poor prognosis. A molecule made during cell metabolism limits metastasis, revealing that this metabolite restrains cancer progression. SEE LETTER P.127

\section{LYDIA W. S. FINLEY}

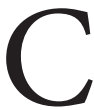
ancer becomes lethal when tumour cells spread from their primary site in the body to invade distant organs - a process termed metastasis. For this complex event to occur, the cells must invade their surrounding tissue, enter the bloodstream and colonize another location, where secondary tumours called metastases form. Several of the early steps in metastasis, including cell migration, can be induced by the abnormal activation of a normal developmental program called epithelial-mesenchymal transition (EMT), in which the epithelial cells that line body surfaces take on the characteristics of mesenchymal cells, which have migratory properties ${ }^{1}$. On page 127 , Wang et al. ${ }^{2}$ identify a previously unknown mechanism by which a molecule generated in cellular metabolism inhibits the induction of EMT and thereby restrains the metastasis of lung cancer in mice.
The molecules formed during metabolism can have a key role in supporting the survival, proliferation and metastasis of tumour cells. Cancer cells have a higher than normal level of nutrient uptake and altered metabolic pathways, and these properties ensure that tumours make the metabolites they need to grow ${ }^{3}$. When tumour cells migrate into the bloodstream, they experience cellular stress. This is characterized by an increase in molecules called reactive oxygen species, and metabolic alterations that counter such stress can promote metastasis ${ }^{3,4}$. But whether metabolic pathways affect other aspects of metastasis has been poorly understood.

To investigate this further, Wang and colleagues individually blocked the expression of 111 metabolic enzymes in human lung cancer cells that had arisen from epithelial cells. Using these cells grown in vitro, the authors found that inhibiting production of the enzyme UGDH impaired the migratory capacity of the

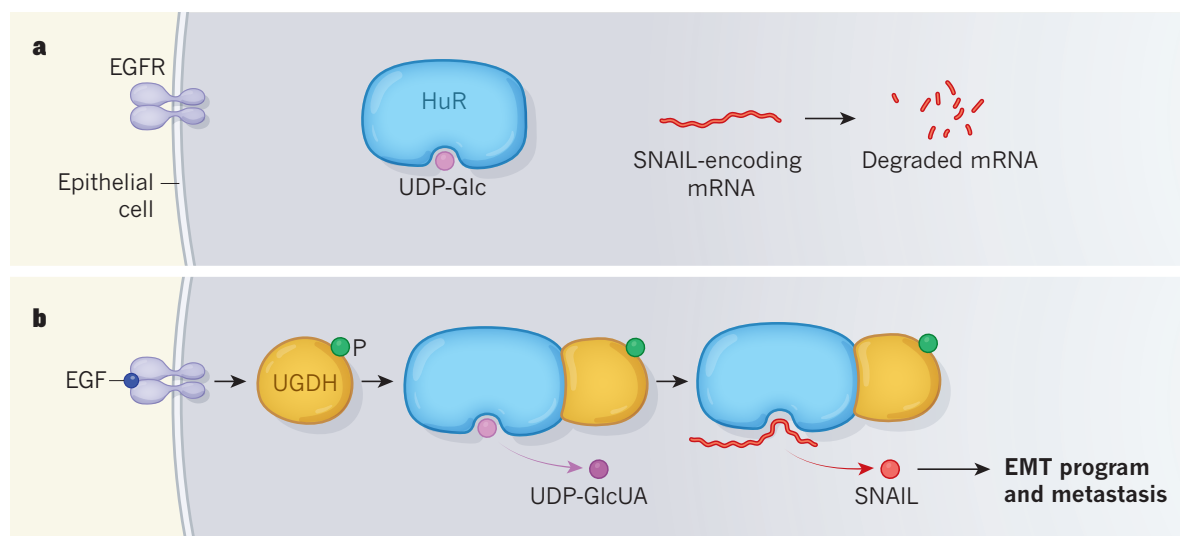

Figure 1 | A molecule formed during cellular metabolism hinders cancer-cell migration. Wang et al. ${ }^{2}$ investigated how metabolism affects the migration (metastasis) of human lung cancer cells grown in vitro or transplanted into mice. These tumours arise from a type of cell called an epithelial cell. a, In epithelial cells that express the receptor EGFR, binding of the protein HuR to a UDP-glucose (UDP-Glc) molecule prevents HuR from binding to and stabilizing the messenger RNA that encodes the SNAIL protein, so that this mRNA is degraded. $\mathbf{b}$, When signalling through EGFR is activated by the EGF protein, a phosphate group $(\mathrm{P})$ is added to the enzyme UGDH, enabling UGDH to bind to HuR. UGDH catalyses the conversion of UDP-Glc to UDP-glucuronic acid (UDP-GlcUA). The authors propose that UGDH carries out this conversion on UDP-Glc bound to HuR, enabling HuR to bind to and stabilize SNAIL mRNA. This allows SNAIL to be produced, facilitating a process called epithelial-mesenchymal transition (EMT), which aids metastasis. 